\title{
Christoph Kleßmann
}

\section{„Führende Klasse“, Sozialpolitik und Egalisierung in der DDR}

Bei der Betrachtung der DDR-Arbeitsgesellschaft stellt sich zwangsläufig die Frage nach der Bedeutung der Sozialpolitik im Spannungsfeld zwischen proklamierter Egalisierung und faktischer Ungleichheit. Die Sozialpolitik spielte eine Schlüsselrolle, weil hier wichtige Weichenstellungen erfolgten für eine grundsätzliche Veränderung der sozialen Schichtung, aber auch für neue und alte Formen sozialer Differenzierung. Generell geht es um den umfassenden politischen Steuerungsanspruch, den die SED mit ihrer marxistisch-leninistischen Ideologie untermauerte. Dieser Steuerungsanspruch mußte jedoch erst in einem langen Prozeß gegen vielerlei $W_{1}$ derstände durchgesetzt werden. Er traf auf divergierende Interessen und auf ein gesellschaftliches Beharrungsvermögen, dessen Ausschaltung erhebliche Konflikte produzierte. Der Blick auf die „führende Klasse“ läßt das Kernproblem von Egalisierung und Ungleichheit in seiner spezifisch sozialistischen Variante besonders gut erkennen. Die Arbeiterschaft war gleichermaßen Träger und Adressat einer Sozialpolitik, die von den Anfängen der SED bis zum Ende der DDR in zeitlich unterschiedlicher Ausformung und mit wechselnder politischer Gewichtung immer als umfassende Gesellschaftspolitik verstanden wurde.

In den ersten Nachkriegsjahren fungierte Sozialpolitik primär noch als Nothilfe gegen die Kriegsfolgelasten. Hier war aber bereits deutlich erkennbar, daß ihr vorrangiges Ziel die Wiederherstellung der Arbeitsfähigkeit und die Bindung an den Arbeitsplatz war ${ }^{1}$. Mit dem Umbau von Wirtschaft und Gesellschaft sollte der traditionelle Begriff von Sozialpolitik überflüssig werden. Als „Lazarettstation für die Opfer des Kapitalismus“ zählte diese zu den Schlacken der bürgerlichen Periode und galt als verpönt ${ }^{2}$. Unabhängig von solchen offiziösen Begriffsentwicklungen erhielt Sozialpolitik in der DDR als umfassende Gesellschaftspolitik zwei charakteristische Kennzeichen: eine starke Betriebszentrierung, die auch die kulturelle Massenarbeit einschloß, und den flächendeckenden Ausbau des FDGB-Apparats als "Implementationsbürokratie"3 der Sozialpolitik. Offenkundig war das Mißverhältnis zwischen parteioffizieller Ideologie und Staatspraxis. Während der Begriff randständig blieb, spielte die praktische Sozialpolitik eine zentrale Rolle. Der umfassenden Durchsetzung der Planwirtschaft lag jedoch die Vorstellung zugrunde, mit der Vollbeschäftigung könne man die materiellen und kulturellen Bedürfnisse der Gesellschaft im wesentlichen planen und erfüllen, so daß eine eigenständige Sozialpoli-

1 Vgl. Schmidt, Sozialpolitik, S. 698.

2 Ebenda, S. 696.

3 Hockerts, Grundlinien Sozialpolitik, S. 523. 
tik überflüssig würde ${ }^{4}$. Mit den sechziger Jahren begann eine neue Phase, in der der Begriff auch in seinen traditionellen Konnotationen eine Renaissance erfuhr und in der sich die intensiven Diskussionen darauf konzentrierten, wie die notwendigen Leistungsanreize mit der sozialen Abfederung der Modernisierungsfolgen der Wirtschaftsreform verbunden werden könnten ${ }^{5}$. Diese zum Teil sehr nüchternen Bilanzen in internen Debatten waren zweifellos eine Reaktion auf die Erfahrungen der fünfziger Jahre und somit auch ein gewisser Lerneffekt. Daß dieser nicht zum Zuge kommen konnte, war wohl weitestgehend systembedingt. Mit Beginn der Ära Honecker wurde die Sozialpolitik zum Markenzeichen einer neuen Periode. Ihre Definition erfuhr eine so extensive Erweiterung, daß sie alle Konturen verlor. Sozialpolitik umfaßte demnach „alle Maßnahmen, Mittel und Methoden, die direkt oder indirekt die Tätigkeit oder das Leben der Bürger berühren. " 6

Die DDR-Geschichte ist insbesondere in ihrer Frühphase unter der Leitfrage nach „Sowjetisierung“ gedeutet worden. Für die zeitgenössischen Publikationen der fünfziger Jahre schien diese Richtung politisch evident zu sein, in der neueren Historiographie hat der Begriff eher eine heuristische Funktion? ${ }^{7}$. Er versucht, das komplexe Geflecht von politischen Intentionen und davon oft beträchtlich abweichender gesellschaftlicher Realität zu erhellen. Die Sozialpolitik ist ohne Zweifel ein markantes Feld, auf dem das Sowjetisierungs-Paradigma an deutliche Grenzen stößt und starke deutsche Traditionsbestände erkennbar werden ${ }^{8}$. Das betrifft Konzepte, Organisation, Personal, aber auch Erwartungen insbesondere der „führenden Klasse“ an ihre selbsternannte Avantgarde. Die sozialstrukturelle Entwicklung der frühen DDR ist von dem gleichen Dualismus geprägt. Die politischen Transformationsvorgaben und der Wille zum Aufbau einer „Gegengesellschaft" zur Bundesrepublik ${ }^{9}$ bildeten ohne Zweifel den Motor der tiefgreifenden Veränderungen, zugleich aber gab es spezifische Rücksichtnahmen auf die eigene Ausgangssituation und die prekäre Lage eines sozialistischen Teilstaats mit offener Grenze zum „Klassenfeind“ im Westen. Das heutige Bild von der frühen DDR-Gesellschaft wird daher nicht mehr so ausschließlich wie früher von dem lautstark betonten Willen zur revolutionären Veränderung bestimmt, sondern berücksichtigt auch stärker die noch vorhandenen „bürgerlichen Relikte“, auf die der „Arbeiter-und-BauernStaat" aus ökonomischen Gründen nicht verzichten konnte.

Die zentralen Merkmale des nach sowjetischem Vorbild modellierten Gesamtsystems lassen sich mit wenigen Hinweisen grob charakterisieren.

1. Kern der sozialistischen Revolution sollte die Abschaffung der Ausbeutung des Menschen sein. Das wichtigste Instrument dafür bildete die Umgestaltung der Eigentumsverhältnisse in Industrie und Landwirtschaft. Die DDR blieb dennoch auch im Selbstverständnis - abgesehen von der Episode von Ulbrichts „sozialistischer Menschengemeinschaft" - eine nichtantagonistische Klassengesellschaft.

4 Schmidt, Sozialpolitik, S. 695.

5 Vgl. Hübner, Sozialpolitische Denk- und Handlungsfelder, in: Geschichte der Sozialpolitik in Deutschland seit 1945, Bd. 9 (Kap. II), erscheint voraussichtlich 2003.

6 Politisches Wörterbuch, S. 1073.

7 Vgl. Lemke, Sowjetisierung; Jarausch/Siegrist, Amerikanisierung.

8 Hockerts, S. 9.

9 Vgl. Jarausch, Gegengesellschaft. 
2. In der neuen Wirtschaftsverfassung wurde die Bourgeoisie als Klasse abgeschafft. An ihre Stelle trat der auf neuen Fundamenten ruhende Staat als ökonomisches Lenkungsinstrument, dem sich auch die noch bestehenden Reste eines Mittelstandes weitgehend zu unterwerfen hatten.

3. Das Dogma der führenden Rolle einer Partei im System von Massenorganisationen und verbündeten Parteien sicherte die Konzentration und Monopolisierung der politischen und sozialen Entscheidungskompetenz. Ein umfangreicher Sicherheitsapparat stützte dieses Machtmonopol ab. Dieses Grundgerüst blieb, auch wenn in der Praxis die „Grenzen der Diktatur" 10 immer wieder sichtbar wurden.

4. Durch vielfältige und gezielte Förderung der Arbeiter und Bauern, verbunden mit Diskriminierung und tendenzieller Ausschaltung bürgerlicher Schichten und Gruppen, sollte die „führende Klasse“ so entwickelt werden, daß sie ihren Anspruch auf politische Führung tatsächlich einlösen konnte.

5. Erwerbsarbeit bildete den Mittelpunkt der Arbeitsgesellschaft, und der Betrieb rückte in historisch neuer Form ins Zentrum sozialer und kultureller Aktivitäten. Er wurde zu einem herausgehobenen Element der beruflichen Lebenswirklichkeit und auch der privaten Alltagserfahrung.

6. Das ideologische Muster bildete zwar die Sowjetunion. Ihre Rolle war und blieb jedoch prekär, da das ideologische Leitbild durch die individuelle und kollektive Erfahrung von Eroberung, Besatzung und Intervention überlagert und immer wieder in Frage gestellt wurde. Die DDR besaß daher als „besetzter Verbündeter" 11 nur wenig Spielraum, den es freilich jeweils genau auszuloten gilt, und war viel stärkeren Zwängen zur strikten Unterordnung als die Bundesrepublik ausgesetzt.

Der Anspruch, ein „Arbeiterstaat“ zu sein, leitete sich neben dem Vorbild der Sowjetunion aber auch aus der Tradition der sozialistischen Arbeiterbewegung ab. Er ließ sich - wenn überhaupt - nur einlösen, wenn der Begriff Arbeiter weit verstanden wurde. Diese legitimatorisch unverzichtbaren Bemühungen begannen bereits in den vierziger Jahren und hielten mit verschiedenen Variationen, die oft ebenso gekünstelt wie diffus waren, bis zum Ende der DDR an. In der formativen Phase der späten vierziger und frühen fünfziger Jahre bemühte sich die SED besonders nachdrücklich darum, den prinzipiellen sozialen Wandel in der politischen und staatlichen Elite zu dokumentieren, indem sie tendenziell zwei Arbeiterbegriffe entwickelte. Christoph Boyer hat sie, obwohl die Quellen diese klare Differenzierung nicht konsequent spiegeln, für das kaderpolitische Programm der SED analysiert ${ }^{12}$. Demzufolge wurde unterschieden nach "Herkunft aus der Arbeiterklasse“, das heißt beruflichem Aufstieg bereits vor 1945, und „Zugehörigkeit zur Arbeiterklasse vor dem Eintritt in den Staatsapparat", das heißt Aufstieg aus den Betrieben in politische und administrative Funktionen in der Entstehungs- und Gründungsphase der DDR. Aufschlußreich für die sozialstatistische Verwirrung durch ideologische Setzungen ist der Hinweis des Mitglieds des ZK der SED, Anton Ackermann, der 1949 im Zusammenhang mit der Diskussion um die Arbeiter-und-Bauern-Fakultäten von einem erweiterten Arbeiterbegriff ausging und feststellte: „Das ist eigentlich

10 Vgl. Bessel/Jessen, Grenzen der Diktatur.

11 Dieser für die frühe Bundesrepublik verwandte Begriff trifft in anderer Akzentuierung auch auf die DDR zu. Rupieper, Der besetzte Verbündete.

12 Boyer, Arbeiterkarrieren? 
selbstverständlich, wird aber nicht immer genügend beachtet. Ich bin auch seit 1929 aus dem Betriebe weg und gehöre doch zur Arbeiterklasse, und wenn ich einen Sohn oder ein Mädchen in dem Alter für das Hochschulstudium hätte, würde ich für meine Kinder beanspruchen, daß sie als Arbeiterkinder gezählt werden; denn ein Parteifunktionär, der im Angestelltenverhältnis in der Partei oder in der Verwaltung steht, ist ein Funktionär seiner Klasse und gehört in erster Linie zu dieser Klasse. In diesem Punkte müssen wir also ganz fest und klar sein. "13 Zur Klarheit dürfte diese Äußerung kaum beigetragen haben, die politisch-ideologische Expansion des Begriffs wird darin jedoch plastisch faßbar.

Die sozialstrukturellen Wandlungen folgten auch in der DDR dem generellen Trend von Industriegesellschaften, aber mit deutlicher Verzögerung: ein schrumpfender Primärsektor, die Expansion des Sekundärsektors mit einem anschließenden allmählichen Rückgang, schließlich das durch die gezielte Industriepolitik der SED verzögerte Anwachsen des Dienstleistungsbereichs. Ideologisch mußte dabei die Arbeiterklasse führend bleiben, obwohl sie im engeren Sinne langfristig wie überall schrumpfte. Äußerlich wurde dieses delikate Problem durch die statistische Zwangsfusion von Arbeitern und Angestellten seit 1960 gelöst. Damit degenerierte der Begriff „Arbeiterklasse“ endgültig zu einem ideologisch hoch aufgeladenen und sozialgeschichtlich inhaltsleeren Etikett.

Gleichwohl bleibt zu fragen, welche konkreten sozial-, bildungs- und rechtspolitischen Folgen die doppelte Berufung der SED auf das sowjetische Vorbild und auf die Arbeiterbewegungstradition bei der Stilisierung der Arbeiter zur "führenden Klasse" hatte. Diese für das Verständnis der politischen und gesellschaftlichen Entwicklung der DDR zentrale Frage läßt sich nicht mit dem Hinweis auf eine von oben oktroyierte Ideologie beiseite schieben. Vielmehr entfaltete diese Ideologie eine eigene soziale Dynamik mit für die SED-Spitze durchaus unerwünschten Konsequenzen. "In der DDR wurde der Typus des deutschen Facharbeiters politisch umworben und sozial konserviert“, hat Peter Hübner betont. „Er stand in engem Zusammenhang mit einer Wirtschaftspolitik, die weitgehend an den vorhandenen industriellen Strukturen festhielt und diese bis Mitte der sechziger Jahre durch eine kräftig ausgeweitete Grundstoffindustrie untermauerte. "14 Diese Entwicklung war gewollt und politisch gesteuert. Auf diese Weise stieß aber die politische Dispositionsfreiheit der SED ungewollt bereits früh an selbst geschaffene Grenzen. Denn die Kontinuität im industriellen Ambiente verstärkte die Erwartungen der "führenden Klasse" nach einem angemessenen, gerechten Lohnsystem und Lebensniveau und schränkte den Spielraum für sozialökonomisch notwendige Veränderungen auf Kosten der Arbeiterschaft von vornherein erheblich ein. Der 17. Juni 1953 wurde für diese nie aufgelöste Grundkonstellation zum politischen Menetekel. Dieses Schlüsselproblem aller kommunistischen Systeme in Europa erhielt in der DDR besondere Brisanz, weil sie den Vorposten zum kapitalistischen Westen bildete und neben der Tschechoslowakei - der einzige Staat mit einer starken sozialistischen Arbeiterbewegungstradition war ${ }^{15}$.

13 Zit. nach Schneider, Bildung, S. 27.

14 Hübner, Das Jahr 1961, S. 32.

15 Das Folgende im Anschluß an meine Skizze: Arbeiter im „Arbeiterstaat“. 
Die von Jeffrey Kopstein formulierte These vom stillschweigenden „Sozialkontrakt " $z$ wischen politischer Führung und Arbeiterschaft ${ }^{16}$ besitzt eine hohe Erklärungskraft für die lang anhaltende relative Stabilität der DDR. Dieser informelle Sozialvertrag wurde bereits vor dem exzessiven Ausbau der Sozialpolitik in der Ära Honecker wirksam und gehört zur Gründungsgeschichte der DDR. Der aus der Arbeiterbewegung stammende Egalitarismus entfaltete sich in den ersten Nachkriegsjahren besonders nachdrücklich und blieb als Strukturproblem in der frühen DDR prägend. Er war nach dem Aufstand vom 17. Juni kaum mehr zu beseitigen, zumal er partiell der ideologischen Grundorientierung der SED nach revolutionärer Gesellschaftsveränderung entsprach. Er verband sich jedoch - und das war ein Spezifikum der exponierten Lage der DDR - mit einer starken Westorientierung und der Erwartung, auf anderen und gerechteren Wegen zu ähnlichem Wohlstand wie die Bundesrepublik zu gelangen. Da die stimulierende und disziplinierende Funktion eines kapitalistischen Arbeitsmarkts für die Löhne mit der Verstaatlichung der Industrie entfiel, entwickelte sich dieser Egalitarismus zu einer stillschweigend gültigen sozialen Norm. Zu verändern war sie in einer Mangelgesellschaft nur mit hohem politischen Risiko, das die SED-Führung nach den Erfahrungen von 1953 und später nach den zahlreichen Krisen in Polen offenbar nicht mehr einzugehen wagte. Ein interessantes Beispiel dieser Krisenrezeption bietet die Äußerung Otto Grotewohls gegenüber den Leitern der Auslandsvertretungen der DDR im Januar 1957: „Der Ausgangspunkt in Polen ist der Aufstand in Poznan im Juni vorigen Jahres gewesen. Aber der Ausgangspunkt für uns ist der 17. Juni 1953. Und seit dem 17. Juni 1953 haben wir viel gelernt und gebranntes Kind scheut das Feuer. "17

Die fünfziger Jahre waren neben der Aufstandserfahrung noch stark geprägt von einem nicht nur aufgesetzten Pathos des Aufbruchs in eine neue sozialistische Gesellschaft. Mit dem Scheitern des „Neuen Ökonomischen Systems der Planung und Leitung“, dem Generationswechsel und der sich vertiefenden statt abnehmenden Kluft im Entwicklungsniveau zwischen Ost und West machten sich zunehmend Gleichgültigkeit und Interesse am sozialen Status quo breit. Arbeiter nahmen unter den gegebenen politischen Bedingungen ihren Staat beim Wort und prägten der Gesellschaft ihre Interessenstruktur auf. „Wie die Partei in die ,Klasse“ hineinrief“, hat Hartmut $Z_{w a h r}$ festgestellt, „so schallte es heraus. So entstand ein ritualisierter Dialog, den die Beteiligten bis zum Ende der DDR durchhielten. "18

Angesichts der diffusen Ausdehnung des Begriffs „Arbeiterklasse" hat Ina Merkel für den Verzicht auf den Begriff Arbeiter zugunsten der Bezeichnung „Werktätige“ plädiert. „Im Begriff des Werktätigen, unter dem im Verlauf der DDR-Geschichte alle sozialen Schichten subsumiert wurden, spiegelte sich die Allgemeinsetzung täglicher, zeitlich bemessener Arbeit für alle Schichten der Bevölkerung wider. Lebenslange abhängige Erwerbsarbeit definierte den Tages- und Wochenrhythmus der gesamten DDR-Bevölkerung, strukturierte Lebensläufe und formte Geschlechterbeziehungen und Familienzusammenhänge. Zum anderen prägte die Abhängigkeit von der Arbeit als der einzig möglichen Quelle des Erwerbs finanzieller Mittel das Verhältnis zu Eigentum, zu ökonomischem Gewinn, zu Aufstieg und Distink-

$16 \mathrm{Vgl}$. Kopstein, Shipping.

17 Zit. Lemke, Einheit oder Sozialismus, S. 385.

$18 \mathrm{Zwahr}$, Arbeitermilieus, S. $10 \mathrm{f}$. 
tion. [...] Dies alles mußte zu einer Annäherung von Lebenslagen führen, zumal die auf Angleichung zielende Lohn- und Preispolitik dafür die materiellen Ausgangsbedingungen zu schaffen suchte. " ${ }^{19}$ In eine ähnliche Richtung hat Wolfgang Engler argumentiert mit seiner Wortschöpfung der „arbeiterlichen Gesellschaft“. „Es wäre eine Absurdität zu behaupten, die ostdeutschen Arbeiter hätten die politische Herrschaft ausgeübt. Aber das soziale Zepter hielten sie in der Hand. Anschauungen, Meinungen, Konventionen, Kleidungs- und Konsumgewohnheiten und nicht zuletzt die Alltagssitten richteten sich nach den Normen und Idealen der arbeitenden Klasse. " 20

So umstritten diese These insgesamt sein mag, so gibt sie doch unzweifelhaft einen wichtigen Teilbefund wieder. Sie verweist auf die tiefgreifende soziale Umschichtung und die Verwischung tradierter Grenzen im Sozialstatus. Eine umfassende Ausprägung fand dieser Prozeß erst seit den sechziger Jahren, die Grundlagen aber wurden bereits vorher gelegt.

Nach der Monopolisierung der politischen Macht durch die SED sind vor allem zwei Instrumente zu nennen, mit denen die Konturen dieser ,arbeiterlichen Gesellschaft" realisiert wurden: die „Brechung des bürgerlichen Bildungsmonopols“ und die Etablierung des FDGB als größter Massenorganisation und Implementationsbürokratie für eine umfassende Gesellschaftspolitik.

Die Forderung nach Abbau sozialer Schranken und besonderer Förderung traditionell benachteiligter Schichten konnte zunächst auf ein breites Echo rechnen. Sie gehörte neben Sozialisierung und Planwirtschaft bei Kriegsende überall zu den Postulaten, die einen gesellschaftlichen Neuaufbau signalisieren sollten. Die Vorstudienanstalten, die 1949 in die „Arbeiter-und-Bauern-Fakultäten“ (ABF) umgewandelt wurden, waren neben der Einführung der Einheitsschule der sichtbarste institutionelle Ausdruck dieses bildungspolitischen Neuansatzes. Dabei verschmolzen in beiden Fällen deutsche Traditionen aus der Reformpädagogik der Weimarer Republik mit sowjetischen Vorbildern. Die Absolventen der ABF sollten zum wichtigsten Rekrutierungspotential einer neuen sozialistischen Intelligenz werden. Darüber hinaus aber bestimmte in den fünfziger Jahren eine geradezu schematische Festlegung des Arbeiter- und Bauernanteils - proportional zur Bevölkerungsschichtung - die Politik der Zulassung zu weiterführenden Schulen ${ }^{21}$.

So eindeutig und folgenreich das politische Programm der „Brechung des bürgerlichen Bildungsmonopols" war, so schwierig ist die Frage zu beantworten, wie weit und in welchen Formen dieses Angebot zum sozialen Aufstieg durch gezielte Privilegierung von der Arbeiterschaft akzeptiert wurde und wie stark der Effekt einer sozialistischen Kaderrekrutierung tatsächlich ausfiel. Zumindest macht die Frühgeschichte der Bildungspolitik in der DDR das Dilemma deutlich, daß kulturelle Grenzen des Sozialmilieus auch unter neuen politischen Bedingungen nicht leicht aufzubrechen waren, um solche Angebote wirksam werden zu lassen. Sonja Häder hat in mikrohistorischen Untersuchungen exemplarisch verdeutlicht, wie stark die Milieugrenzen als Barrieren für einen schnellen Erfolg dieser Bildungsoffensive

19 Vgl. Merkel, Arbeiter und Konsum, S. 543 f.

20 Vgl. Engler, Die Ostdeutschen, S. $198 \mathrm{f}$.

$21 \mathrm{Vgl}$. Anweiler, Schulpolitik. 
noch waren ${ }^{22}$. Aufstieg durch Bildung gehörte zu den alten Forderungen und Hoffnungen der sozialistischen Arbeiterbewegung. Ihre Umsetzung stieß aber in Teilen der Arbeiterschaft immer wieder auf Ängste vor den Folgen einer Entfremdung gegenüber dem vertrauten Milieu. Zweifellos gelang der SED im Vergleich zur Bundesrepublik relativ schnell und dauerhaft ein viel höheres Maß sozialer Egalisierung, und zwar durch Privilegierung der Unterschichten ebenso wie durch zeitweilig konsequente Diskriminierung von Familien alter Eliten. Generationsspezifisch verschoben sich aber seit den sechziger Jahren die Erfahrungs- und Verhaltensmuster, wie Annegret Schüle am Beispiel von Textilarbeiterinnen gezeigt hat ${ }^{23}$. Während die Aufbaugeneration mit katastrophalen Kindheitserfahrungen ihren relativ gesicherten Ort in der DDR-Gesellschaft ihrem Betrieb und dem Aufstieg im Betrieb verdankten, besaß die jüngere Generation von Frauen einen ganz anderen Erfahrungshintergrund. Sie war in relativer sozialer Sicherheit groß geworden, hatte ein eher pragmatisches Verhältnis zu den Angeboten des Staates und einen kritischeren Blick für seine Mißstände. Als Reaktion auf die pflichtbewußte, opferbereite Müttergeneration im Betrieb ließen sich bei den Jüngeren eher Formen von Aufstiegsverweigerung finden. Diese Befunde sind zwar nicht ohne weiteres generalisierbar, sie verweisen aber auf eine charakteristische Gemengelage unterschiedlicher Faktoren in der Einstellung von Arbeitern wie Dankbarkeit, Überforderungsängste und auch Gleichgültigkeit.

Ein widersprüchliches Bild zeigt sich auch dort, wo Arbeiterinteressen traditionell am ehesten organisiert waren: in den Gewerkschaften. Der FDGB ist zeitgenössisch und historiographisch zu Recht als wichtigste Massenorganisation und "Transmissionsriemen“ des Parteiwillens entsprechend Lenins Konzept wahrgenommen und dargestellt worden. Mittlerweile ist jedoch deutlicher erkennbar geworden, daß die Spannung zwischen dem sowjetischen Modell und deutscher Tradition, zwischen dem FDGB als Träger von Kampagnen zur Steigerung der Produktivität und als berufliche und sozialpolitische Interessenorganisation von Lohnabhängigen im engeren Sinne nie vollständig verschwand ${ }^{24}$. Insofern weist die Geschichte des FDGB unter der Oberfläche eine merkwürdige Ambivalenz auf. Seine tatsächliche soziale Rolle deckte sich keineswegs vollständig mit der ihm zugedachten politischen Funktion. Er hat offenbar, wie Peter Hübner festgestellt hat, auch eine Art „Krypto-Geschichte“ gehabt, die starke Verbindungslinien zum stets bekämpften "Sozialdemokratismus" und zum sogenannten "Nur-Gewerkschaftertum" besaß. Die eine Linie war eng an die SED-Spitze gebunden und vor allem im Bundesvorstand präsent. „Die andere hatte ihre Wurzeln in der Gewerkschaftsbewegung der Zwischenkriegszeit, mit starken Querverbindungen zur Sozialdemokratie, und sie blieb längerfristig in der Funktionärsschicht der Industriegewerkschaften sowie unter den vorwiegend ehrenamtlichen Betriebsfunktionären erhalten. Vor allem von dieser Seite her kam ein deutlicher pragmatischer Zug, der sich insbesondere im sozialen Aufgabenbereich des FDGB Geltung verschaffte. " 25

22 Vgl. Häder, Schülerkindheit.

23 Vgl. Schüle, Mächtige Mütter.

${ }^{24}$ Für die Anfangsphase besonders aufschlußreich: Werum, „Wir sind die Illegalen!“.

25 Hübner, Der FDGB, S. 33. 
Aufgrund seiner Schlüsselrolle im Betrieb waren Arbeiter ständig mit dem FDGB konfrontiert: Er fungierte als Motor der ständig eingeforderten Produktionssteigerung, er war aber auch Träger betrieblicher Sozialpolitik und Kulturarbeit, also von unmittelbar und individuell erfahrbaren sozialen, gesundheitlichen und kulturellen Leistungen. Insofern produzierte er neben Reibungen und Konflikten auch beträchtliche politische Loyalitätseffekte für das Regime, auch wenn sein Image an der Basis der Belegschaften überwiegend verheerend war. Positive Integrationswirkungen gingen vor allem von den Brigaden als „Herzstück“ der Arbeitsorganisation in der DDR aus. Obwohl kollektive Arbeitsorganisation kein Spezifikum realsozialistischer Systeme war, trugen die 1947 erstmals eingeführten Arbeitsbrigaden, vor allem in ihrer 1959 geschaffenen „veredelten“ Form als „Brigaden der sozialistischen Arbeit “, doch unverwechselbare Züge. Hier kreuzten sich interessenpolitische und lebensweltliche Bezüge. Wie neuere Studien nachgewiesen haben $^{26}$, ist die Geschichte der Brigaden daher zu einem nicht unerheblichen Teil auch eine verdeckte oder offene Konfliktgeschichte. Da die Gewerkschaften ihre traditionelle Funktion als Interessenorganisation kaum erfüllen konnten, verlagerten sich die Erwartungen auf die Brigaden - nicht ohne Erfolg, wie Umfragen zu unterschiedlichen Zeiten belegen. In einer zeitgenössischen Untersuchung von 1957 zur Einstellung geflüchteter Arbeiter aus großen Industriebetrieben wird der Brigade ein gutes Zeugnis ausgestellt ${ }^{27}$. Obwohl von der SED propagiert, wurde sie als Leistungseinheit weitgehend akzeptiert. Ferner zeigten Gespräche mit den Arbeitern deutlich, daß als "Errungenschaften des Sozialismus" vor allem die mannigfaltigen sozialen Einrichtungen in den Betrieben verstanden wurden. Solche sozialgeschichtlichen Differenzierungen im früher überwiegend politisch geprägten Bild von der größten Massenorganisation verändern nicht grundsätzlich die tragenden Elemente in der "politischen Konstruktion des Sozialen“ 28 , aber sie verweisen auf eine konstitutive Spannung, die Helke Stadtland auf die treffende Formel gebracht hat: „Herrschaft nach Plan und Macht der Gewohnheit“29.

Der Konflikt zwischen proklamierter Egalisierung und ökonomisch notwendiger Leistungsdifferenzierung und daraus resultierender sozialer Ungleichheit wurde nie aufgelöst, er war aber in den fünfziger Jahren besonders wirksam, weil der Verzicht auf bürgerliche Fachleute noch nicht möglich und der gesellschaftliche Umbau nicht im gewünschten Tempo realisierbar schien. Insgesamt ist dennoch die soziale Pyramide in der DDR frühzeitig deutlich flacher geworden als im Westen, sieht man von besonderen Privilegien für Spezialisten mit Einzelverträgen, Nationalpreisträgern und speziellen Gratifikationen ab. Ein Kernproblem bestand in der Konstellation, die Kopstein als "geheimen Sozialvertrag“ zwischen Arbeiterklasse und Führung charakterisiert hat. Diese Situation resultierte einerseits aus der ideologisch erzwungenen Rücksichtnahme der SED auf die „führende Klasse“ und war andererseits ein Ergebnis der Schockerfahrung des Aufstandes vom 17. Juni. Beides zusammen hat Sozialpolitik als Gesellschaftspolitik nachhaltig geprägt. Die ideolo-

26 Vgl. Roesler, Produktionsbrigaden. Soldt, Schwarze Pumpe. Die Dissertation von Thomas

Reichel zu den sozialistischen Brigaden ist in Vorbereitung.

$27 \mathrm{Vgl}$. Blücher, Industriearbeiterschaft.

28 Tenfelde, Einige Ergebnisse, S. 889.

29 Stadtland, Herrschaft. 
gische Privilegierung der breit definierten Arbeiterschaft bestimmte zunehmend das soziale Profil der Gesamtgesellschaft, ohne jemals eine politische Herrschaft der

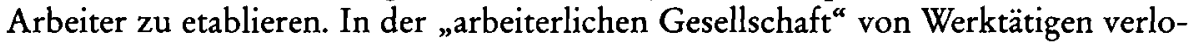
ren traditionelle Schichtungskriterien ihre alte Bedeutung. Sie wurden aber zunehmend durch neue "feine Unterschiede" ersetzt, die weniger sichtbar, aber durchaus wirksam und schwerer zu steuern waren. 
